Ethik Med 2006 · 18:223-237 DOl 10.1007/s00481-006-0439-5 Online publiziert: 16. August 2006 ○) Springer Medizin Verlag GmbH 2006
Hans-Jörg Ehni, Urban Wiesing · Tübingen

\section{Placebos in klinischen Versuchsreihen}

\author{
Eine vergleichende Analyse \\ der internationalen Richtlinien
}

\section{Einleitung}

Klinische Forscher und Ethiker debattieren seit dem letzten Jahrzehnt ausführlich und kontrovers über die ethischen Aspekte der Placebo-Kontrolle in klinischen Versuchen. In diesem Zusammenhang haben mehrere internationale Organisationen wie der Weltärztebund und der Council for International Organizations of Medical Sciences ihre Richtlinien zur biomedizinischen Forschung revidiert. Während viele Autoren die Deklaration von Helsinki (DoH) und ihre neueste Änderung zum Gebrauch von Placebo („Note of Clarification", 2002) bis ins Detail untersucht und kritisiert haben $[8,14,16,17,23,25]$, erhielten andere internationale Richtlinien wie diejenigen der CIOMS oder der International Conference on Harmonisation weniger Aufmerksamkeit. Eine gründliche, vergleichende Analyse der verschiedenen internationalen Richtlinien, ihrer Terminologien und ihrer ethischen Prinzipien in Bezug auf die Placebo-Kontrolle fehlt zudem vollständig. Der vorliegende Artikel möchte einen Beitrag zu einer solchen Analyse leisten, indem er die ethischen Regelwerke der World Medical Association (WMA), des Council for International Organizations of Medical Sciences (CIOMS), der International Conference on Harmonisation of Technical Requirements for Registration of Pharmaceuticals for Human Use (ICH), von UNAIDS miteinander vergleicht. Als internationales Regelwerk mit rechtlicher Verbindlichkeit für die unterzeichnenden und ratifizierenden Nationen beziehen wir ferner das Zusatzprotokoll zur Konvention von Oviedo des Europarats in die Analyse ein (Additional Protocol to the Convention on Human Rights and Biomedicine, concerning Biomedical Research, in der Folge als „Forschungsprotokoll des Council of Europe" abgekürzt) [6]. Zusätzlich werden die relevanten Teile der Stellungnahmen der European Group on Ethics (EGE) [26], des Nuffield Council [22] und des National Bioethics Advisory Committee [21] berücksichtigt.

Die anhaltende Kontroverse bezieht sich vor allem auf die Rechtfertigung einer Placebo-Kontrolle, wenn eine nachgewiesen wirksame Therapie existiert. Die gegenwärtigen Fassungen aller untersuchten Richtlinien sehen eine solche Rechtfertigung vor, jedoch in unterschiedlicher Form. Daher stehen die Bedingungen, unter denen jeweils die Verwendung von Placebo ethisch erlaubt ist, im Mittelpunkt dieser Untersuchung. Zunächst werden wir ein formales, allgemeines Prinzip herausarbeiten, das die ethische Angemessenheit der Verwendung von Placebo definiert. Im Anschluss werden wir drei Kategorien von Bedingungen analysieren, die die verschiedenen Regelwerke als Rechtfertigungen anführen: das minimale Risiko eines Schadens oder eine minimale Belastung, zwingende wissenschaftliche Gründe und die mangelnde Erhältlichkeit nachweislich wirksamer Therapien. Die Analyse zeigt bedeutende normative Abweichungen und Widersprüche zwi- 
schen den untersuchten Richtlinien auf. Besonders frappierend ist der Umstand, dass manche Richtlinien es erlauben, die Versuchsteilnehmer dem Risiko eines schwerwiegenden Schadens auszusetzen, während andere gerade dies untersagen. Schließlich wollen wir zeigen, wie die verschiedenen normativen Positionen der einzelnen Richtlinien die Entscheidung von Forschern und Ethikkommissionen darüber beeinflussen können, ob es ethisch angemessen ist, eine Placebo-Kontrolle zu verwenden.

\section{Das allgemeine Prinzip}

Wenn die Placebo-Kontrolle in klinischen Versuchen bei einer nachgewiesen wirksamen Therapie die Ausnahme darstellen soll, muss zunächst die Regel definiert werden, d.h. vor allem muss die Qualität der aktiven Kontrolle und damit das Behandlungsniveau der Kontrollgruppe festgelegt werden. Die gegenwärtige Fassung der Deklaration von Helsinki verwendet z. B. die Formulierung „best current prophylactic, diagnostic, and therapeutic methods" ${ }^{1}$. Andere Formulierungen wie „established effective intervention " wurden vorgeschlagen und zum Gegenstand einer weiteren intensiv geführten Debatte. Diese wiederzugeben, würde den Umfang des vorliegenden Artikels sprengen. Es ist jedoch möglich, unabhängig davon welche Qualität der aktiven Kontrolle als angemessen definiert wird, nur die Bedingungen zu untersuchen, unter denen diese durch ein Placebo ersetzt werden darf. Die Definition der angemessenen aktiven Kontrolle wird nur dann Teil des Placebo-Problems, wenn bereits eine bestimmte Formulierung als solche im Vergleich zu anderen eine weniger restriktive Praxis impliziert. Dies ist beispielsweise der Fall, wenn die Formulierung „local available treatment" anstelle von „best current treatment" verwendet wird. Tatsächlich kann diese Formulierung auf eine der vor-

\footnotetext{
1 Da es von den maßgeblichen Texten der internationalen Richtlinien in der Regel keine autorisierte deutsche Übersetzung gibt und es hier auf den Wortlaut ankommt, behalten wir die englischen Originalfassungen bei.
}

geschlagenen Bedingungen bezogen werden, unter der eine nachweislich wirksame Therapie als möglicher Komparator durch ein Placebo ersetzt werden kann. Wir werden darauf im entsprechenden Teil dieses Artikels eingehen. ${ }^{2}$ Im Übrigen reicht es für die Zwecke dieses Artikels aus, die jeweils vorgeschlagenen Formulierungen zur aktiven Kontrolle durch X zu ersetzen. Dementsprechend lautet eine allgemeine Regel für die ethische Angemessenheit eines klinischen Versuchs im Bezug auf die Verwendung von Placebo wie folgt:

Ein klinischer Versuch ist ethisch erlaubt, wenn die Kontrollgruppe als Komparator eine Therapie erhält, die X entspricht, oder wenn anstelle von $\mathrm{X}$ ein Placebo verwendet wird und die Bedingungen a-n erfüllt sind.

Dies veranschaulicht der relevante Artikel 29 der Deklaration von Helsinki: „The benefits, risks, burdens and effectiveness of a new method should be tested against those of the best current prophylactic, diagnostic, and therapeutic methods. This does not exclude the use of placebo, or no treatment, in studies where no proven prophylactic, diagnostic or therapeutic method exists."

Ersetzt man die Variablen in Prinzip (o) durch den entsprechenden Wortlaut der Deklaration von Helsinki, erhält man folgendes Prinzip $\left(1^{\star}\right):\left(1^{\star}\right)$ Ein klinischer Versuch ist ethisch erlaubt, wenn die Kontrollgruppe als Komparator eine Therapie erhält, die den „best current prophylactic, diagnostic, and therapeutic methods" entspricht, oder wenn ein Placebo verwendet wird und keine "proven prophylactic, diagnostic, and therapeutic method" existiert.

Der Deklaration von Helsinki und allen anderen Richtlinien zufolge ist die Verwendung von Placebo ethisch legitim, sofern keine Therapie existiert, welche die Funktion eines Komparators erfüllen könnte. Das allgemeine Prinzip kann daher so formuliert werden:

\footnotetext{
${ }^{2}$ Vgl. den Abschnitt: Die Erhältlichkeit einer nachgewiesen wirksamen Therapie als Kriterium für die Verwendung von Placebo.
} 


\section{Zusammenfassung $\cdot$ Abstract}

Ethik Med $2006 \cdot 18: 223-237$

DOI 10.1007/s00481-006-0439-5

C) Springer Medizin Verlag GmbH 2006

Hans-Jörg Ehni, Urban Wiesing

\section{Placebos in klinischen Versuchsreihen. Eine vergleichende Analyse der internationalen Richtlinien}

\section{Zusammenfassung}

Die ethischen Aspekte der Verwendung von Placebos in klinischen Versuchsreihen wurden im letzten Jahrzehnt ausführlich und kontrovers diskutiert. Es fehlt dennoch eine gründliche vergleichende Analyse der verschiedenen internationalen Richtlinien, ihrer Terminologien und ihrer auf Placebo bezogenen Prinzipien. Das zentrale Problem ist die Rechtfertigung von Placebo bei einer nachgewiesen wirksamen Therapie. Alle aktuellen Versionen der untersuchten Richtlinien schlagen solche Rechtfertigungen vor, unterscheiden sich hierbei jedoch beträchtlich. Zunächst werden wir ein formales allgemeines Prinzip herausarbeiten. Dann werden wir drei verschiedene Kategorien von Kriterien darstellen: das Schadensrisiko oder die Belastung, zwingende wissenschaftliche Gründe und die Erhältlichkeit einer nachgewiesen wirksamen Therapie. Die Analyse zeigt bedeutende normative Abweichungen und Widersprüche innerhalb und zwischen den einzelnen Richtlinien. Besonders frappierend ist es, dass manche Richtlinien es erlauben, Versuchsteilnehmer dem Risiko eines schweren Schadens auszusetzen, während andere gerade dies untersagen. Abschließend versuchen wir zu zeigen, wie sich die normativen Unterschiede bei der Entscheidungsfindung von Forschern und Ethikkommissionen auswirken können.

\section{Schlüsselwörter}

Placebo - Klinische Versuchsreihen . internationale Richtlinien . Deklaration von Helsinki . internationale Forschung

\section{Placebos in clinical trials. A comparative analysis of international guidelines}

\section{Abstract}

Definition of the Problem: The ethical aspects of placebo control in clinical trials have been extensively and controversially debated in the last decade. However, a thorough analytical comparison of the different existing international regulations, their terminologies and their ethical principles concerning placebo is still missing. The central issue in the ongoing controversy is the justification of placebo-use if proven treatment exists. All present versions of the examined guidelines propose such justifications, but each guideline differs from the others in relevant details. Therefore the conditions justifying placebo-use according to each guideline are in the focus of our attention.

Arguments: First, we will first present a formalized general principle that defines the ethical acceptability of placebo use in all regulations. Then we will analyse three categories of conditions put forward by the different documents: the risk of harm or burden, compelling scientific reasons, and the availability of proven treatment. The analysis shows important normative discrepancies and contradictions between the examined guidelines. Especially striking is the fact that some guidelines allow to expose the participants of clinical trials to be exposed to a risk of serious harm, while others do not.

Conclusion: The normative difference of each guideline could influence strongly the decision of researchers or IRBs concerning the ethical acceptability of placebo use.

\section{Keywords}

Placebo - Clinical trials .

International guidelines - Declaration of Helsinki . International research 
(1) Ein klinischer Versuch ist ethisch erlaubt, wenn die Kontrollgruppe als Komparator eine Therapie erhält, die X entspricht, oder wenn ein Placebo verwendet wird und keine Therapie existiert, die $\mathrm{X}$ entspricht.

Abgesehen von verschiedenen Formulierungen für $\mathrm{X}$, ist das Prinzip (1) allen folgenden ethischen Richtlinien gemeinsam und unumstritten:

- Declaration of Helsinki: Art. 29 s.o. [28].

- CIOMS: Placebo may be used: „when there is no established effective intervention" (Guideline 11) [5].

- ICH: „When a new treatment is tested for a condition for which no effective treatment is known, there is usually no ethical problem with a study comparing the new treatment to placebo“ (2.1 Placebo Control) [12].

- Council of Europe: „The use of placebo is permissible where there are no methods of proven effectiveness" (Art. 23, 3) [6].

- UNAIDS: „As long as there is no known effective HIV preventive vaccine, a placebo control should be ethically acceptable in a phase III HIV preventive vaccine trial (Guidance Point 11)“ [13].

- EGE: „In the absence of any existing treatment, the comparison can be carried out with a placebo" $[26,1.4]$.

Es besteht eine weite Übereinkunft über dieses allgemeine Prinzip und darüber, dass Ausnahmen gerechtfertigt werden müssen. Man kann außerdem festhalten, dass die Formulierungen der International Conference on Harmonisation und der Deklaration von Helsinki vorsichtiger sind. Sie lauten, dass „üblicherweise“ („usually“) (ICH) die Verwendung von Placebo keine ethische Problematik darstellt, wenn keine Therapie bekannt ist oder dass die Verwendung von Placebo in solchen Fällen nicht „ausgeschlossen“ („excluded“) sei (DoH), ohne dies weiter auszuführen. Andere Richtlinien bekräftigen schlicht in diesem Zusammenhang: „placebo may be used“ (CIOMS); oder: „it is permissible" (Council of Europe).

\section{Ausnahmen vom allgemeinen Prinzip}

Das allgemeine Prinzip (1) wird mittlerweile in allen internationalen Richtlinien oder Empfehlungen durch verschiedene Bedingungen für Ausnahmen ergänzt, während einige Kritiker seine strenge, ausnahmslose Gültigkeit befürworten [1, 11, 23]. Als Bedingungen für die Ausnahmen werden folgende Kriterien genannt: wissenschaftliche Gründe, eine minimale Belastung oder minimales Risiko eines schweren oder irreversiblen Schadens für die Patienten und die fehlende Erhältlichkeit nachgewiesen wirksamer Therapien. Entsprechend können die folgenden Versionen von (1) formuliert werden, die angeben, in welchen Fällen die Verwendung von Placebo trotz nachgewiesener Therapie ethisch erlaubt ist.

(1.1) Ein klinischer Versuch mit Placebo-Kontrolle ist auch bei nachgewiesen wirksamer Therapie ethisch erlaubt, sofern nur eine minimale Belastung oder minimales Risiko eines schweren oder irreversiblen Schadens für die Versuchsteilnehmer bestehen.

(1.2) Ein klinischer Versuch mit Placebo-Kontrolle ist auch bei nachgewiesen wirksamer Therapie ethisch erlaubt, sofern aus wissenschaftlichen Gründen eine Placebo-Kontrolle erforderlich ist.

(1.3) Ein klinischer Versuch mit Placebo-Kontrolle ist auch bei nachgewiesen wirksamer Therapie ethisch erlaubt, sofern die nachgewiesen wirksame Therapie in dem Land oder in der Region nicht erhältlich ist, in dem oder in der der Versuch stattfindet.

Im Folgenden seien die zusätzlichen Kriterien hinsichtlich der unterschiedlichen Terminologien, Formulierungen und im Blick auf mögliche Kombinationen untereinander untersucht. 


\section{Das "Schadensrisiko" oder die „Belastung" als Kriterien für die Verwendung von Placebo}

(1.1) Ein klinischer Versuch mit PlaceboKontrolle ist auch bei nachgewiesen wirksamer Therapie ethisch erlaubt, sofern nur eine minimale Belastung oder minimales Risiko eines schweren oder irreversiblen Schadens für die Versuchsteilnehmer bestehen.

Das Kriterium „Schadensrisiko“, das Prinzip (1.1) zugrunde liegt, ist allen Richtlinien gemeinsam. Dagegen verwendet nur das Forschungsprotokoll des Council of Europe explizit „burden“ als verwandtes, zusätzliches Kriterium zur Begrenzung des möglichen Schadens. Die Formulierungen "temporary discomfort" oder "delay in the relief of symptoms" der CIOMS-Richtlinien können daneben lediglich als implizite Beispiele für eine minimale Belastung interpretiert werden. Die ICH Richtlinie bezieht sich auf „serious harm“, ohne nähere Spezifikation. In anderen Richtlinien fehlt der Bezug auf das grundlegende Kriterium des "Schadensrisikos“ völlig.

Viele Kommentatoren betrachten ein minimales Schadensrisiko oder eine minimale Belastung als akzeptable Kriterien für die ethische Rechtfertigung von Placebo, wenn eine nachgewiesen wirksame Therapie existiert. Dies ist auch wenig umstritten, wenngleich Rothman und Michels argumentieren, dass weder ein einzelner Forscher noch eine einzelne Ethikkommission festlegen sollte, welche Risiken oder Belastungen für die Teilnehmer eines klinischen Versuchs minimal sind. Sollten einzelne Personen oder Institutionen das Recht haben, darüber zu entscheiden, würde das beiden Autoren zufolge dazu führen, dass vulnerable Personengruppen weniger vor Missbrauch geschützt sind. Bereits aus diesem Grund sollte ihrer Meinung nach eine nachgewiesen wirksame Therapie niemals in der Kontrollgruppe eines klinischen Versuchs durch ein Placebo ersetzt werden [19].
Im Gegensatz zu dieser Position ist die Verwendung von Placebo nach der Note of Clarification zu Artikel 29 der Deklaration von Helsinki dann ethisch legitim, wenn eine neue Behandlungsmethode für einen nicht schwerwiegenden Zustand gesucht wird oder wenn die Patienten keinem zusätzlichen Risiko eines schweren oder irreversiblen Schadens ausgesetzt sind (,a new method is investigated for a minor condition and the patients who will receive placebo will not be subject to any additional risk of serious or irreversible harm"). Beispiele dafür fehlen, was "minor condition“ oder was "serious or irreversible harm" bedeuten können.

Die Richtlinien des CIOMS beinhalten die sorgfältigste Formulierung beider Kriterien und eine zusätzliche Bedingung, was in dieser Kombination nirgendwo sonst gefunden werden kann. Zunächst ist demnach die Verwendung von Placebo unter der folgenden Vorbedingung ethisch erlaubt: „when withholding an established effective intervention would expose subjects to, at most, temporary discomfort or delay in relief of symptoms“. Die Begriffe "temporary discomfort" oder "delay in relief of symptoms" präzisieren, welche Belastung oder welcher Schaden annehmbar ist. Der Kommentar zur entsprechenden Richtlinie 11 führt ebenfalls Beispiele an: "slightly raised blood pressure“ oder "a modest increase in serum cholesterol" werden als Risiken eines geringen Schadens eingestuft; eine "common cold“ oder "hair loss" werden als "minor conditions" angesehen, und "common headache" wird als annehmbarer "temporary discomfort" bezeichnet.

Zusätzlich zu dieser klaren Definition der Annehmbarkeit eines Risikos oder einer Belastung, ergänzen die CIOMS-Richtlinien das Kriterium „wissenschaftliche Gründe“. Nur wenn die Verwendung von Placebo ebenfalls aus „zwingenden und wissenschaftlich schlüssigen methodischen Gründen“ („,compelling and scientifically sound methodological reasons") erforderlich ist, ist sie ethisch erlaubt. Da 
beide Bedingungen gleichzeitig erfüllt sein müssen, damit Ausnahmen von der allgemeinen Regel zulässig sind, ist dies eine restriktivere Regulierung als bei den übrigen Richtlinien.

Die einschlägigen Formulierungen des Council of Europe und der ICH sind unbestimmter und führen lediglich „unacceptable risk or burden“ bzw. "serious harm“ als Ausschlusskriterien für eine PlaceboKontrolle an, sofern eine nachgewiesen wirksame Therapie existiert. Das Forschungsprotokoll des Council of Europe erlaubt es nur in solchen Fällen eine nachgewiesen wirksame Therapie vorzuenthalten, wenn sowohl Risiko als auch Belastung annehmbar sind und überlässt die präzise Definition der beteiligten Ethikkommission und der zuständigen Körperschaft („ethics committee and competent body"). Die Position der ICH ist demgegenüber weniger restriktiv: einschränkendes Kriterium für Placebo-Kontrollen ist nur ein schwerwiegender Schaden, wie z. B. Tod oder unumkehrbare Morbidität (,serious harm, such as death or irreversible morbidity“ 2.1.3). Akzeptabel wäre beispielsweise ein Versuch mit kurzer Dauer, in dem ein neues Mittel zur Blutdrucksenkung bei Bluthochdruckpatienten ohne Erkrankung der Endorgane erprobt wird („a short term trial of a new antihypertensive agent in patients with essential hypertension and no end-organ disease"), während das „wahrscheinlich“ nicht der Fall sei bei länger andauernden Versuchen oder solchen, an denen schwerer erkrankte Patienten teilnehmen. Der Ausdruck „wahrscheinlich“ zeigt an, dass die Definition der „Annehmbarkeit“ zu einem sehr großen Maß einem Dialog zwischen Patienten, Forschern und Institutional Review Boards (IRB) bzw. independent ethics committees (IEC) überlassen wird. Als Ausnahme sei zudem eine Placebo-Kontrolle trotz des Risikos eines schwerwiegenden Schadens ethisch erlaubt, wenn zahlreiche Patienten eine nachgewiesen wirksame Therapie aufgrund ihrer Toxizität ablehnen. Die Annehmbarkeit eines
Schadensrisikos - so die ICH - sei überdies vom lokalen oder regionalen Kontext abhängig.

Diese nähere Untersuchung zeigt also im Bezug auf die Kriterien „risk of harm“ und "minimal burden“ bedeutende Unterschiede zwischen den einzelnen Richtlinien auf. Die Richtlinie 11 des CIOMS ist in dieser Hinsicht am restriktivsten, während die Deklaration von Helsinki eine mittlere Position einnimmt. Das Forschungsprotokoll des Council of Europe und die Richtlinien des ICH legen es nahe, die Entscheidung über die Annehmbarkeit eines Risikos oder einer Belastung im Einzelfall dem Forscher und der verantwortlichen Ethikkommission zu überlassen. Während alle Richtlinien darin übereinstimmen, dass die Verwendung von Placebo ethisch erlaubt ist, wenn nur minimales Risiko eines schweren Schadens besteht, bleibt die folgende Frage offen: Kann eine PlaceboKontrolle selbst dann gerechtfertigt werden, wenn Versuchsteilnehmer einem solchen Risiko ausgesetzt sind?

\section{Zwingende wissenschaftliche Gründe zur Rechtfertigung von Placebo-Kontrollen}

Es geht also um die folgende Ausnahme für die Zulässigkeit einer Placebo-Kontrolle:

(1.2) Ein klinischer Versuch mit Placebo-Kontrolle ist auch bei nachgewiesen wirksamer Therapie ethisch erlaubt, sofern aus wissenschaftlichen Gründen eine Placebo-Kontrolle erforderlich ist.

Wie im vorherigen Abschnitt gezeigt wurde, sieht man in der Regel das minimale Risiko eines schweren Schadens oder eine minimale Belastung als Kriterien für die Verwendung von Placebo an. Kann demgegenüber eine hinreichende Bedingung für eine Placebo-Kontrolle auf wissenschaftlichen Gründen beruhen, gleichgültig ob das Risiko eines schweren Schadens besteht oder mehr als nur eine leichte Belastung? Die gegenwärtige Version der Deklaration von Helsinki scheint diese Fra- 
ge mit "Ja" zu beantworten, weshalb sie scharf kritisiert wurde. Ihre Formulierung lautet:

- „[...] a placebo-controlled trial may be ethically acceptable, even if proven therapy is available, under the following circumstances: Where for compelling and scientifically sound methodological reasons its use is necessary to determine the efficacy or safety of a prophylactic, diagnostic or therapeutic method;“ (Note of Clarification to Article 29).

Andere Richtlinien führen ebenfalls wissenschaftliche Gründe für die ethische Legitimität von Placebo an, schließen jedoch z.T. jedes erhöhte Risiko eines schweren Schadens aus. Sie lauten wie folgt:

- CIOMS: „[...] when use of an established effective intervention as comparator would not yield scientifically reliable results [Hervorhebung durch die Autoren] and use of placebo would not add any additional risk of serious or irreversible harm to the subjects." (Guideline 11).

- UNAIDS: „Participants in the control arm of a future phase III HIV preventive vaccine trial should receive an HIV vaccine known to be safe and effective when such is available, unless there are compelling scientific reasons which justify the use of a placebo." (Guidance 11).

\section{Die „Note of Clarification“ zu Art. 29 der Deklaration von Helsinki}

Demzufolge schließt die „Note of Clarification" der Deklaration von Helsinki eine Placebo-Kontrolle auch bei Risiko eines schweren Schadens nicht aus. Manche Kritiker sahen darin die Konsequenz einer falsch gewählten logischen Verknüpfung [2]. Es sei inakzeptabel - und auch nicht intendiert gewesen -, die Bedingung des
Risikos eines schweren Schadens und die Bedingung, die auf wissenschaftlichen Gründen beruht, mit dem logischen Operator „oder“ $\mathrm{zu}$ verknüpfen. Denn aufgrund dieser logischen Verknüpfung muss nur eine dieser beiden Bedingungen erfüllt sein. Andere Kommentatoren sehen in dieser Festlegung einen Widerspruch zum Prinzip, dass die Interessen des Individuums Vorrang gegenüber den Interessen von Wissenschaft und Gesellschaft besitzen sollen (und folglich auch einen Selbstwiderspruch in der Deklaration von Helsinki, im Bezug auf Art. 5) [20]. Die auf diese Weise kritisierte Position der Note of Clarification lässt sich in folgendem Prinzip wie folgt wiedergeben:

(1.2.1) Ein klinischer Versuch mit Placebo-Kontrolle ist auch bei nachgewiesen wirksamer Therapie ethisch erlaubt, sofern aus wissenschaftlichen Gründen eine Placebo-Kontrolle erforderlich ist und eine schwerwiegende Belastung oder das Risiko eines schweren oder irreversiblen Schadens für die Versuchsteilnehmer besteht.

Gleichgültig ob dies ein unbeabsichtigter logischer Fehler ist oder nicht: es trifft nicht $\mathrm{zu}$, dass niemand die in (1.2.1) formulierte logische Konsequenz auch tatsächlich vertritt [15]. Mindestens die UNAIDS-Richtlinien und auch einige Kommentatoren [3, 7] rechtfertigen explizit die Verwendung von Placebo mit zwingenden wissenschaftlichen Gründen trotz des Risikos eines schweren Schadens und einer schwerwiegenden Belastung.

\section{Was sind „zwingende" wissenschaftliche Gründe und welches Risiko oder welche Belastung können sie rechtfertigen?}

Da jede Versuchsanordnung wissenschaftlichen Kriterien genügen muss, kommt es auf den Zusatz „zwingend“ an. Es ist möglich, die Placebo-Kontrolle mit wissenschaftlichen Gründen zu rechtfertigen, die nicht zwingend sind, weil eine alternative Versuchsanordnung ebenfalls wissenschaftlich erfolgreich sein kann. Daher 
halten wir das Kriterium „compelling scientific reason" nicht deswegen für redundant, weil ohnehin jeder klinische Versuch wissenschaftlich einwandfrei sein müsse, wie Ruth Macklin meint [18]. Wenn eine wirksame Therapie nachgewiesen ist, müssen gerade „zwingende“ Gründe für eine Placebo-Kontrolle angeführt werden können, die gleichzeitig jede andere mögliche Kontrollgruppe einer alternativen Versuchsanordnung ausschließen. Der „Note of Clarification“ zufolge muss der Forscher nicht nur nachweisen, dass wissenschaftliche Gründe die Verwendung von Placebo rechtfertigen, sondern dass diese notwendig ist und nicht durch einen anderen Kontrolltyp ersetzt werden kann.

Vorausgesetzt, eine solche Rechtfertigung sei möglich, was könnten dann solche „compelling and scientifically sound methodological reasons" sein, wie sie die „Note of Clarification“ verlangt? Welche Art von Notwendigkeit ist gemeint? Die Deklaration von Helsinki führt keine weitere Erklärung oder Beispiele an. Eine genauere Untersuchung des Problems erlauben dagegen die Richtlinien von UNAIDS, die entsprechende Beispiele für eine Verknüpfung von wissenschaftlichen Gründen und dem Risiko eines schweren Schadens enthalten. Wenn eine nachweislich wirksame und sichere HIV-Impfung erhältlich wäre, dann ließen sich folgende wissenschaftliche Gründe für eine Placebo-Kontrolle zur Entwicklung eines weiteren Impfstoffes anführen:

- Die Überzeugung, dass eine zukünftig entwickelte, üblicherweise wirksame HIV-Impfung keine Wirkung bei dem Virustyp zeigen wird, der in der Personengruppe vorherrscht, zu der die Versuchsteilnehmer gehören.

- Der Umstand, dass die biologischen Bedingungen des Versuchs, mit dem die Wirksamkeit des vorhandenen Impfstoffs nachgewiesen wurde, sich erheblich von denjenigen der Personengruppe unterscheiden, aus der die Versuchsteilnehmer stammen; die
Resultate des ursprünglichen Versuchs ließen sich in diesem Fall nicht übertragen [13, Guideline 11].

In beiden Beispielen basieren die „methodologischen“ Gründe auf der Hypothese, dass die vorhandene Therapie als aktive Kontrolle keine verlässliche Bezugsgröße darstellt und unter den gegebenen besonderen Bedingungen nicht wirksam sein wird.

Können dieser Standpunkt von UNAIDS und folglich ebenfalls die „Note of Clarification" gegen ihre Kritiker mit Hilfe dieser Beispiele verteidigt werden, wenn sie das Risiko eines schweren Schadens erlauben? Können diese Beispiele tatsächlich so verstanden werden, dass sie "Zwingende“ wissenschaftliche Gründe beinhalten?

Die angeführten Beispiele von UNAIDS beruhen nicht nur auf wissenschaftlichen, methodologischen Gründen, sondern sie sind implizit auch auf den möglichen Nutzen für die Versuchsteilnehmer bezogen, was in der "Note of Clarification" nicht der Fall ist. Zusätzlich zur Bedingung, dass der Versuch ohne Placebo verlässliche Resultate hervorbringen würde, kann die Bedingung aufgestellt werden, dass die Teilnehmer keinen Nutzen aus der vorhandenen Therapie ziehen würden. Zwei kritische Anmerkungen lassen sich gegen diese Beispiele anführen:

Sofern die Befürworter einer PlaceboKontrolle argumentieren, eine nachgewiesen wirksame Therapie würde keine verlässliche Bezugsgröße darstellen, müssen sie ebenfalls die generellen Zweifel gegenüber Placebo als Referenz in Betracht ziehen [13, 27]. Manchen Kritikern zufolge ist Placebo im Allgemeinen keine bessere Vergleichsgröße als eine aktive Kontrolle mit einer nachgewiesen wirksamen Therapie, da der Placebo-Effekt gleichfalls eine große Variabilität unter verschiedenen Bedingungen aufweist [11, S. 241]. So weist ein klinischer Versuch möglicherweise nach, dass ein neues, billigeres Medikament einem Placebo überlegen ist. Aber unter der Voraussetzung unterschiedlicher biologi- 
scher Bedingungen, lassen sich die Wirksamkeit der getesteten Therapie und der bereits vorhandenen Therapie nicht mittels der Placebo-Kontrolle vergleichen.

Zweitens könnte als Argument für eine Placebo-Kontrolle angeführt werden, dass die Versuchsteilnehmer nicht von der nachgewiesen wirksamen Therapie profitieren, weil gleichzeitig noch das Risiko unerwünschter Nebenwirkungen vorhanden ist. Dies ist ein entscheidender Aspekt, denn er widerspricht der Auffassung, dass bei einer Placebo-Kontrolle aus wissenschaftlichen Gründen unzulässigerweise die Interessen von Wissenschaft und Gesellschaft über die Interessen des Individuums stellt.

Beide Argumente, gleichgültig ob sie sich auf Placebo als Bezugsgröße oder auf den Nutzen der Teilnehmer stützen, beruhen jedoch letztlich auf der Grundannahme, dass die nachgewiesen wirksame Therapie unter den Bedingungen eines geplanten Versuchs und in der entsprechenden Personengruppe, aus der die Teilnehmer stammen, einem Placebo nicht überlegen ist. Dies entspricht dem elementaren wissenschaftlichen und ethischen Kriterium der „clinical equipoise“, d.h. der genuinen Unsicherheit bezüglich der Qualität zweier verschiedener Behandlungsmethoden [9, S. 141; 10, S. 253]. In diesem Fall muss eine "clinical equipoise" hinsichtlich einer bereits nachgewiesenen Behandlungsmethode und einem Placebo vorliegen, ungeachtet des Umstands, dass bereits unter anderen Bedingungen gezeigt wurde, dass diese einem Placebo überlegen ist. Gerade aber diese, bisherigen Erkenntnissen zunächst widersprechende Annahme wird ohne einen weiteren Vergleich der Behandlungsmethode mit Placebo schwierig zu belegen sein. Folglich wird keine empirische Evidenz für die genannte Grundannahme vorhanden sein, und es kann sein, dass diese sich als spekulative Voraussetzung entpuppt und keine ausreichende wissenschaftliche und ethische Grundlage für die Verwendung von Placebo darstellt. Gerade der Nachweis der Annahme, dass eine ge- nuine Unsicherheit bestehe, ob die zu testende Behandlungsmethode Placebo überlegen sei, wird nun zum Problem. Während beispielsweise auch Placebo im vorliegenden Fall andere Resultate als zuvor liefert, wäre es möglich, dass eine nachgewiesen wirksame Therapie sich zwar unter den vorliegenden Bedingungen als weniger wirksam erweist, aber immer noch einem Placebo überlegen ist.

Folglich muss zwar die Rechtfertigung von Placebo durch „zwingende“ wissenschaftliche Gründe nicht immer dem Prinzip widersprechen, das die Interessen des Individuums hervorhebt. Aber die wissenschaftliche Notwendigkeit von Placebo sollte im einzelnen Fall sorgfältig geprüft werden. Auch bei der Anwendung der CIOMS-Richtlinie steht man vor demselben Problem, selbst wenn das zusätzliche Risiko eines schweren oder irreversiblen Schadens ausgeschlossen wird.

\section{Modifikationen der Versuchsanordnung: die ICH-Richtlinie}

Eine andere Möglichkeit, dem Risiko eines schweren Schadens zu begegnen, wird von der Richtlinie der ICH vorgeschlagen, die keine allgemeinen Regeln aufstellt, wann z.B. eine Placebo-Kontrolle auszuschließen sei. Sie zählt lediglich die normativen und wissenschaftlichen Vor- und Nachteile der verschiedenen Kontrolltypen auf, zu denen Placebo, keine Behandlung oder aktive Kontrolle gehören. Jeder dieser Kontrolltypen besitzt bestimmte wissenschaftliche und ethische Vorteile. Die Autoren der ICH-Richtlinie betrachten zunächst Placebo als eine von mehreren gleichermaßen wissenschaftlich gerechtfertigten Optionen für die Wahl der Kontrollgruppe. Bei Placebo bestünden die Vorteile z. B. darin, dass ein entsprechender klinischer Versuch voraussichtlich weniger Teilnehmer benötigt, um aussagekräftig zu sein, als jeder andere Typ einer kontrollierten Studie [12, 2.1.6.3].

Dem allgemeinen Ansatz der ICH zufolge ist Placebo für einen Forscher, der vor 
der Entscheidung steht, welche Art von Kontrollgruppe er wählen soll, immer eine Option. Ein Algorithmus „Choosing the Concurrent Control for Demonstrating Efficacy“ [12, Guideline 10, S. 29] fasst dies deutlich zusammen. Er zeigt, wie ein Forscher bei der Wahl zwischen den verschiedenen Kontrollmöglichkeiten vorgehen muss. Der Wissenschaftler muss einige Fragen der Reihe nach beantworten, wobei „Ja“ oder „Nein“ zur nächsten Frage weiterführt oder zu verschiedenen Optionen für die Versuchsanordnung. Wird der vollständige Algorithmus durchlaufen, muss folgendes beantwortet werden: Frage 1: „Is there proven effective treatment?", Frage 2: „Is the proven effective treatment life saving or known to prevent irreversible morbidity“, und Frage 3: „Is there historical evidence of sensitivity to drug effects for an appropriately designed and conducted trial“. In diesem Zusammenhang ist die Antwort auf Frage 2 von besonderem Interesse. Selbst wenn diese Frage mit "Ja“ beantwortet wurde, bleibt eine Placebo-Kontrolle immer noch eine Option für den Forscher. In diesem Fall muss die Versuchsanordnung so modifiziert werden, dass das Risiko eines schwerwiegenden Schadens angemessen berücksichtigt wird, z.B. durch den Abbruch des Versuchs für bestimmte Teilnehmer. Hier ist die Verwendung von Placebo also mit dem Risiko eines schweren Schadens verbunden, das zwar durch die Maßnahmen des Forschers eingeschränkt werden soll, nichtsdestotrotz aber von Anfang an präsent ist. Daher muss sich diese Herangehensweise mit derselben Kritik auseinandersetzen wie die Deklaration von Helsinki: die wissenschaftliche Notwendigkeit einer Placebokontrolle muss im Einzelfall belegt werden und gleichzeitig muss der Nachweis geführt werden, dass die Interessen des Individuums nicht denjenigen von Wissenschaft oder Gesellschaft geopfert werden.

\section{Die Erhältlichkeit einer nachgewiesen wirksamen Therapie als Kriterium für die Verwendung von Placebo}

Ist eine nachgewiesene, wirksame Therapie in einem Land oder einer Region nicht erhältlich, so legitimiert dies für manche Forscher oder Ethiker eine Placebo-Kontrolle. Darauf beruht ein drittes Prinzip für die Verwendung von Placebo, das wie folgt formuliert werden kann:

(1.3) Ein klinischer Versuch mit Placebo-Kontrolle ist auch bei nachgewiesen wirksamer Therapie ethisch erlaubt, sofern die nachgewiesen wirksame Therapie in dem Land oder der Region nicht erhältlich ist, in dem oder der der Versuch stattfindet.

\section{Die Reaktion auf die HIV-Versuche von 1997}

Die Kontroverse um Placebo wurde von klinischen Studien ausgelöst, die Mitte der 9oer Jahre in verschiedenen Entwicklungsländern stattfanden, z.B. in Thailand, Äthiopien und anderen afrikanischen Ländern. Die Studien sollten eine Therapie testen, welche die HIV-Übertragung von der Mutter auf das Kind während der Schwangerschaft verhindern sollte. Diese Therapie sollte weniger aufwendig und billiger als die bereits vorhandene sein, damit das neue Mittel für Patienten in Entwicklungsländern erschwinglich und $\mathrm{zu}$ gänglich sein würde. Die werdenden Mütter in den Kontrollgruppen erhielten ein Placebo. Die Hauptkritik an dieser Vorgehensweise war, dass die Erhältlichkeit einer nachgewiesen wirksamen Therapie die Versuchsanordnung aus ethischen Gründen nicht beeinflussen sollte.

Das Kriterium „Erhältlichkeit einer nachgewiesen wirksamen Therapie“ ist umstrittener und komplexer als die beiden anderen. Seine Komplexität beruht auf der Verbindung mit zusätzlichen näheren Bestimmungen, die die detaillierten Richtlinien und Kommentare von CIOMS und UNAIDS anführen. Darüber hinaus haben 
die European Group on Ethics, der Nuffield Council, und das National Bioethics Advisory Committee dieses Problem ausführlich in ihren entsprechenden Empfehlungen behandelt. Die Deklaration von Helsinki erwähnt die Erhältlichkeit einer möglichen aktiven Kontrolle nicht explizit als eine Bedingung für die Verwendung von Placebo. Die Formulierung „in absence of existing treatment “ in der "Note of Clarification “ könnte jedoch aufgrund der Ambiguität von „absence“ dahingehend interpretiert werden. Dieser Ausdruck könnte zwar als „non-existence“ verstanden werden, aber „absence of existing treatment" wird man eher als „unavailability of existing treatment" auffassen. Ansonsten hätte man die Formulierung der vorherigen Version beibehalten können: „where no treatment exists“. Die Richtlinien der ICH und das Forschungsprotokoll des Council of Europe bejahen die fehlende Erhältlichkeit als Kriterium der Verwendung von Placebo in jeweils kurzen und einfachen Stellungnahmen. Demzufolge kann die Entscheidung über die Kontrollgruppe von den erhältlichen Medikamenten oder Vorgehensweisen in der jeweiligen Region abhängen („the decision may depend on the available drugs or medical practices in the specific regions", [12, Figure 1]. Oder nach dem Council of Europe: „It is expected that a proven method of treatment that is available in the country or region concerned be utilized [as a treatment provided to the control group ] " 6 , Commentary to Article 23, $\$ 120]$.

Diese Formulierungen verweisen auf die bereits zu Beginn dieses Artikels erwähnte Möglichkeit, X im allgemeinen Prinzip (1) durch „local available treatment" zu ersetzen. Ein entsprechendes Prinzip $\left(1.3^{\star}\right)$ lautet wie folgt:

$\left(1.3^{*}\right)$ Ein klinischer Versuch ist ethisch erlaubt, wenn die Kontrollgruppe als Komparator eine Therapie erhält, die einem „local available treatment" entspricht; oder wenn anstelle eines „local available treatment" ein Placebo verwendet wird und keine Therapie existiert, welche die Funktion als Komparator erfüllen könnte (die Qualität eines "local available treatment" besitzt).

Zwischen (1.3) und (1.3*) gibt es einen bedeutenden ethischen Unterschied, der gegen das letztere Prinzip spricht. Unabhängig davon wie letztlich die ethisch angemessene Qualität von X definiert wird, muss sie auch auf den Prinzipien des Wohltuns (beneficence) und Nicht-Schadens (non-maleficence) beruhen. Wenn bereits eine Formulierung von $\mathrm{X}$ als solche den leicht erreichbaren Nutzen oder Schutz vor Schaden einschränken würde, dann muss diese Konzeption zurückgewiesen werden. Ein ethisches Prinzip zur Regulierung der Verwendung von Placebo, das nur verlangt eine lokal erhältliche Therapie von voraussichtlich geringerer Qualität gegenüber einer global erhältlichen zur Verfügung $\mathrm{zu}$ stellen, widerspricht dem Prinzip des Wohltuns. Wenn z.B. die lokal erhältliche Therapie Injektionsspritzen wiederverwendet, würde ein solches Prinzip den Forscher oder Sponsoren nicht zur Nutzung von Einwegspritzen verpflichten, unbesehen des Aufwands. Folglich wäre ein solches Prinzip ethisch nicht haltbar, wie z.B. Ruth Macklin festgehalten hat [18].

\section{Ausbeutung vermeiden: wie soll das Behandlungsniveau der Kontrollgruppe festgelegt werden?}

Wenn Prinzip $\left(1.3^{*}\right)$ ethisch nicht $\mathrm{zu}$ rechtfertigen ist, stellt sich die Frage, ob dies auch für (1.3) gilt. Wie soll die Erhältlichkeit einer nachgewiesen wirksamen Therapie bei der Verwendung von Placebo berücksichtigt werden? Stützt man sich auf die Erhältlichkeit einer solchen Therapie, kann eine bestimmte Versuchsanordnung in dem Land, in dem der Versuch stattfindet, als ethisch erlaubt betrachtet werden, was jedoch nicht im Herkunftsland des Forschers und des Geldgebers der Fall wäre. Der Komparator kann nur deswegen ein Placebo sein, weil eine nachgewiesene Therapie aus ökonomischen 
oder aus logistischen Gründen nicht erhältlich ist. Folglich wäre eine bestimmte Versuchsanordnung nur aufgrund von Armut ethisch erlaubt - eine offensichtlich sehr problematische normative Konsequenz. Außerdem könnte dieses Prinzip auch zu einem Teufelskreis führen: Wird die Therapie, die sich in entsprechenden Versuchen in Entwicklungsländern als wirksam erwiesen hat, schließlich in Industriestaaten lizenziert und produziert, wäre sie möglicherweise wiederum nicht dort erhältlich, wo sie erprobt wurde [26, 2.10 The use of placebo]. Daher wird die Rechtfertigung von Placebo durch die mangelnde Erhältlichkeit einer Therapie $\mathrm{zu}$ einem Problem, das eng mit dem Risiko der Ausbeutung verknüpft ist, das u.a. durch das niedrige Behandlungsniveau und die weniger strengen Regulierungen in Entwicklungsländern erzeugt wird. Um dies $\mathrm{zu}$ verhindern, haben die Richtlinien von UNAIDS und CIOMS mehrere Zusätze als Bedingungen hinzugefügt, die ergänzend zum Prinzip 1.3 gelten sollen:

(1.3.1) CIOMS Kommentar zur Richtlinie 11: Ein klinischer Versuch mit PlaceboKontrolle ist auch bei nachgewiesen wirksamer Therapie ethisch erlaubt, wenn die nachgewiesene Therapie in dem Land oder in der Region nicht erhältlich ist, in dem der Versuch stattfindet

und

(a) wenn es unwahrscheinlich ist, dass diese Therapie in der näheren Zukunft erhältlich sein wird, und

(b) wenn der Zweck einer solchen Studie darin besteht, für die entsprechende Bevölkerung des Landes, der Region oder der Gemeinschaft eine wirksame Alternative zur nachgewiesen wirksamen, aber nicht erhältlichen Therapie zugänglich zu machen, und

(c) wenn die vorgeschlagene, untersuchte Behandlungsmethode auf die gesundheitlichen Bedürfnisse der Bevölkerung zugeschnitten ist, und

(d) wenn die vorgeschlagene, untersuchte Behandlungsmethode, sofern sie sich als wirksam und sicher erweist, für diese Bevölkerung auf zumutbare Weise zugänglich („reasonably available“) gemacht werden wird, oder

(e) wenn die verantwortlichen wissenschaftlichen und ethischen Kommissionen überzeugt worden sind, dass die nachgewiesen wirksame Behandlungsmethode nicht als Komparator verwendet werden kann, weil auf diese Weise keine verlässlichen wissenschaftlichen Ergebnisse erzielt werden können, die für die gesundheitlichen Bedürfnisse der Studien-Population relevant wären („established effective intervention cannot be used as a comparator because it would not yield scientifically reliable results that would be relevant to the health needs of the study population") [5, Guideline 11, Commentary].

Die zusätzlichen Bedingungen (b), (c), und (d) sollen Ausbeutung vermeiden. Eine neue Therapie soll nicht in einem Entwicklungsland getestet werden, sofern ausschließlich die Bevölkerung im Land des Auftraggebers der Studie von ihr profitiert. Daher muss die klinische Studie die gesundheitlichen Bedürfnisse der jeweiligen Gemeinschaft berücksichtigen, aus der die Teilnehmer der Studie stammen, und die neue Therapie muss ihnen auf zumutbare Weise zugänglich sein. Vor allem darf die Behandlung im Verhältnis zum lokalen Durchschnittseinkommen nicht zu kostspielig werden.

Bei Bedingung (e) bleibt unklar, ob sie mit den übrigen Bedingungen durch eine Konjunktion oder eine Disjunktion verknüpft wird. Der Abschnitt mit dem Titel „Exceptional use of a comparator other than an established effective intervention“ legt es zunächst nahe, diese Verknüpfung als Konjunktion aufzufassen. Aber in diesem Fall wären die Bedingungen (a) bis (d) redundant, weil die mangelnde Erhältlichkeit einer Therapie aus ökonomischen und logistischen Gründen auch mit den entsprechenden Zusätzen keine hinreichende 
ethische Bedingung für eine Placebo-Kontrolle darstellt, während gleichzeitig (e) nur so sinnvoll verstanden werden kann, dass diese Bedingung die Verwendung von Placebo ohne weiteren Zusatz rechtfertigt. Wenn eine aktive Kontrolle keine wissenschaftlich gültigen Ergebnisse erwarten lässt, die für die gesundheitlichen Bedürfnisse der Teilnehmer relevant sind, ist es gleichgültig, ob sie normalerweise erhältlich ist oder nicht. Letztlich kann (e), insofern diese Bedingung eine Placebo-Kontrolle hinreichend rechtfertigen soll, als Anwendung des Prinzips (1.2) betrachtet werden und ist mit denselben ethischen Problemen und der oben erwähnten $\mathrm{Be}$ weislast verbunden. Folglich kann (e) im Kontext der Erhältlichkeit einer nachgewiesen wirksamen Therapie keine Rolle spielen. Lediglich als Zusatz von Prinzip (1.2) kommt dieser Bedingung möglicherweise eine Bedeutung zu.

Noch detailreicher regeln die Richtlinien von UNAIDS die Suche nach einem HIV-Impfstoff in der besonderen Situation, die durch die AIDS-Pandemie und extern finanzierte klinische Studien in Entwicklungsländern geprägt ist. Wie bereits oben erwähnt, beruht die Einschränkung der Verwendung von Placebo in der relevanten UNAIDS-Richtlinie auf zwingenden wissenschaftlichen Gründen im Sinn von (1.2). Andere Richtlinien behandeln die Frage des Nutzens (Guidance Point 2: „Vaccine availability“, Guidance Point 10: „Benefits") und des Behandlungsniveaus (Guidance Point 16: „Care and Treatment").

Die Richtlinien 2 and 10 von UNAIDS stellen die ethische Forderung auf, dass eine Therapie, deren Wirksamkeit in einer Studie nachgewiesen wird, für die Teilnehmer der Studie und ebenfalls für die $\mathrm{Ge}$ meinschaft, zu der sie gehören, auf zumutbare Weise zugänglich gemacht werden soll. Das Prinzip der Gerechtigkeit schreibt eine angemessene Verteilung des Nutzens und der Lasten vor. Ungerecht wäre es, wenn die Teilnehmer an einem klinischen Versuch lediglich die mögliche Belastung durch eine Placebo-Kontrolle $\mathrm{zu}$ tragen hätten, ohne dass ihnen der Nutzen zugute kommt. Die fehlende Erhältlichkeit einer nachgewiesen wirksamen Therapie kann daher eine Placebo-Kontrolle nur rechtfertigen, wenn die begründete Aussicht besteht, dass der Versuch diesen Mangel beseitigen wird.

Die European Group on Ethics konnte sich im Bezug auf diese Frage nach eigenem Bekunden nicht auf einen gemeinsamen Standpunkt einigen und ihre Stellungnahme enthält scheinbar widersprüchliche Aussagen [26]: So kann die Verwendung von Placebo gerechtfertigt werden, wenn eine Standardtherapie aus logistischen Gründen nicht erhältlich ist oder aufgrund der Kosten nicht zugänglich ist (2.10 Placebo). Aber: „When a trial is implemented in a country or a community where patients cannot benefit from the standard treatment because of the cost, it is then up to the sponsor to provide it" (2.12 Supply of treatment during the trials). Wenn demzufolge der Sponsor verpflichtet ist, die Teilnehmer eines Versuchs mit der Standardtherapie zu versorgen, dann reicht die fehlende Erhältlichkeit alleine nicht aus, um Placebo zu rechtfertigen, wie aus $2.10 \mathrm{zu}$ folgen scheint.

Die Empfehlungen des Nuffield Council erinnern in hohem Maß an die von UNAIDS. Ideal wäre demnach ein „universal standard of care“, der als „best current method available anywhere in the world“ definiert wird. Dies würde die Verwendung von Placebo ausschließen, wenn eine nachgewiesene Therapie als Komparator aus ökonomischen Gründen nicht erhältlich ist. Aber zusätzlich wird eine Minimalforderung aufgestellt: „the best intervention available as a part of the national public health system of the host country" $[22$, XV]. Ein bedeutender Unterschied zur UNAIDS-Richtlinie besteht im Bezug auf den Gesundheitstourismus in der Formulierung „als Teil des nationalen Gesundheitswesens" („as a part of the national public health system“). Dadurch könnte die Qualität eines möglichen Komparators noch niedriger angesetzt werden - und die 
Gerechtigkeitsproblematik weiter verschärfen. Wie die UNAIDS-Richtlinie, überlässt der Nuffield Council letztlich die Entscheidung den zuständigen Ethikkommissionen.

Dieser Empfehlung schließt sich das $\mathrm{Na}$ tional Bioethics Advisory Committee an. Danach sollen Forscher und Sponsoren unabhängig von deren Erhältlichkeit die Kontrollgruppe mit einer nachgewiesen wirksamen Therapie versorgen („Researchers and Sponsors should design clinical trials that provide members of any control group with an established effective treatment, whether or not such treatment is available in the host country " $[21, \mathrm{Re}$ commendation 2.2]). Jede Ausnahme hiervon muss gerechtfertigt werden, allerdings werden Beispiele für mögliche Rechtfertigungen nicht genannt.

Wie diese Übersicht zeigt, gibt es eine weitreichende Übereinkunft in den Richtlinien und relevanten Berichten darüber, dass die mangelnde Erhältlichkeit einer nachgewiesenen Therapie eine PlaceboKontrolle unter bestimmten Umständen rechtfertigen kann. Im Allgemeinen wird die Entscheidung in letzter Konsequenz den zuständigen Ethikkommissionen überlassen, teilweise mit zusätzlichen Bedingungen. Gleichwohl teilen nicht alle Kommentatoren oder Ethiker diese Überzeugung - diese Rechtfertigung der Placebo-Kontrolle bleibt hochgradig umstritten.

\section{Schlussfolgerung}

Alle untersuchten Richtlinien legitimieren eine Placebo-Kontrolle, auch wenn eine wirksame Therapie nachgewiesen ist. Doch im Detail präsentieren sie ganz unterschiedlich formulierte und kombinierte Bedingungen.

Was folgt aus diesen Unterschieden zwischen den Richtlinien? Die ethische Bewertung eines konkreten Forschungsprojekts kann sehr unterschiedlich ausfallen, je nachdem welche Richtlinie berücksichtigt wurde. Wenn beispielsweise ein Forscher nach einer angemessenen Versuchsanordnung sucht, obwohl eine wirksame Therapie nachgewiesen ist und das Risiko eines schweren oder unumkehrbaren Schadens vorhanden ist, sofern diese Therapie vorenthalten oder abgesetzt wird, sind folgende Resultate möglich:

1. Nimmt er Bezug auf die Deklaration von Helsinki, wird der Forscher untersuchen, ob die Verwendung von Placebo mit zwingenden und wissenschaftlich schlüssigen methodischen Gründen gerechtfertigt werden kann.

2. Berücksichtigt der Wissenschaftler die CIOMS-Richtlinie, wird er die Verwendung von Placebo ausschließen.

3. Gemäß dem Forschungsprotokoll des Council of Europe wird er der zuständigen Ethikkommission die Entscheidung überlassen, ob das Risiko in diesem Fall akzeptabel ist oder nicht.

4. Richtet der Forscher sich nach der ICHRichtlinie, wird er eine modifizierte Placebo-Kontrolle in Betracht ziehen.

5. Vorausgesetzt eine wirksame HIVSchutzimpfung sei bereits entwickelt worden, würde ein Forscher beim Test eines neuen Impfstoffs nach der UNAIDS-Richtlinie prüfen, ob es wissenschaftliche Belege für die Hypothese gibt, dass der vorhandene Impfstoff in der Personengruppe nicht wirksam ist, zu der die Versuchsteilnehmer gehören.

Die Bandbreite der Richtlinien eröffnet demnach einen großen Entscheidungsspielraum. Wünschenswerter wäre ein einheitlicher internationaler Standard. Ein solcher Standard sollte alle Bedingungen für die ethische Legitimität der Verwendung von Placebo berücksichtigen. Ferner sollte die Terminologie vereinheitlicht werden und eine klare Haltung zur Grenze und Einschränkung der Verwendung von Placebo-Kontrollen sollte entwickelt werden. Die gegenwärtige und fortgesetzte Debatte über Placebo-Kontrollen kann nicht nur als zusätzliches Anzeichen für dieses Erfordernis gesehen werden, son- 
dern auch als Chance, neue Erkenntnisse bei einer Revision der vorhandenen Richtlinien einzubeziehen.

\section{Anschrift \\ Hans-Jörg Ehni}

Institut für Ethik und Geschichte der Medizin

- Bereich Ethik in der Medizin -

Schleichstr. 8

72076 Tübingen

E-Mail: hans-joerg.ehni@uni-tuebingen.de

\section{Literatur}

1. Angell M (1997) The Ethics of Clinical Research in the Third World. N Engl J Med 337:847-849

2. Carlson RV, Boyd KM, Webb DJ (2004) The revision of the Declaration of Helsinki: past, present, and future. Br J Clin Pharmacol 57:695-713

3. Carpenter WT, Appelbaum PS, Levine RJ (2003) The Declaration of Helsinki and Clinical Trials: A Focus on PlaceboControlled Trials in Schizophrenia. Am J Psychiatry 160: 356-362

4. Christakis NA (1992) Ethics are local: Engaging cross-cultural variation in the ethics for clinical research. Soc Sci Med 35:1079-1091

5. Council for International Organizations of Medical Sciences CIOMS in collaboration with the World Health Organization (2002) International Ethical Guidelines for Biomedical Research Involving Human Subjects. Geneva

6. Council of Europe (2005) Additional Protocol to the Convention on Human Rights and Biomedicine, concerning Biomedical Research, Strasbourg, 25.I.2005, Council of Europe Treaty Series - No. 195

7. Derivan AT (2004) The Ethical Use of Placebo in Clinical Trials Involving Children. J Child Adolesc Psychopharmacol $14: 169-174$

8. Doppelfeld (2001) World Medical Association: Struktur und Perspektive. Dtsch Arztebl 98:A-1874

9. Freedman B (1987) Equipoise and the Ethics of Clinical Research. N Engl J Med 317:141-145

10. Freedman B, Glass KC, Wejer C (1996) Placebo Orthodoxy in Clinical Research II. J Law Med Ethics 24:252-259
11. Freedman B, Weijer C, Glass KC (1996) Placebo Orthodoxy in Clinical Research I: Empirical and Methodological Myths. J Law Med Ethics 24:243-251

12. International Conference on Harmonisation of Technical Requirements for Registration of Pharmaceuticals for Human Use (2000) ICH Harmonised Tripartite Guideline. Choice of Control Group and Related Issues in Clinical Trials. E 10

13. Joint United Nations Programme on HIV/AIDS (UNAIDS) (2000) Ethical considerations in HIV preventive vaccine research. Geneva

14. Levine RJ (1998) The 'Best Proven Therapeutic Method' Standard in Clinical Trials in Technologically Developing Countries. J Clin Ethics 9:167-174

15. Lie RK, Emanuel E, Grady C, Wendler D (2004) The standard of care debate: the Declaration of Helsinki versus the international consensus opinion. J Med Ethics 30:190-193

16. Lurie P, Wolfe SM (1997) Unethical Trials of Interventions to Reduce Perianatal Transmission of the Human Immunodeficiency Virus in Developing Countries. N Engl J Med 337:853- 856

17. Macklin R (2001) After Helsinki: unresolved issues in international health research. Kennedy Inst Ethics J 11:17-36

18. Macklin R (2004) Double Standards in Medical Research in Developing Countries. Cambridge University Press, Cambridge

19. Michels KB, Rothmann KJ (1994) The Continuing Unethical Use of Placebo Controls. N Engl J Med 331:394-398

20. Michels KB, Rothmann KJ (2003) Update on unethical use of placebos in randomised trials. Bioethics 17:188-204

21. National Bioethics Advisory Commission (2001) Ethical and Policy Issues in International Research: Clinical Trials in Developing Countries. Bethseda, Maryland

22. Nuffield Council on Bioethics (2002) The ethics of research related to healthcare in developing countries. 0.0.

23. Rothmann KJ, Michels KB, Baum M (2000) For and against. Br Med J 321:442-445

24. Schüklenk U (2000) Protecting the vulnerable: testing times for clinical research ethics. Soc Sci Med 51:969-977

25. Taupitz J (2002) Note of Clarification. Kaum zu verantworten. Dtsch Arztebl 99:A-411

26. The European Group on Ethics in Science and New Technologies to the European Commission (2003) Opinion on the ethical aspects of research in developing countries. Office for Official Publications of the European Communities, Luxembourg

27. Weijer C (1999) Placebo-controlled trials in schizophrenia: Are they ethical? Are they necessary. Schizophr Res 35:211218

28. World Medical Association (2002) Declaration of Helsinki. www.wma.net/e/policy/pdf/17c.pdf 